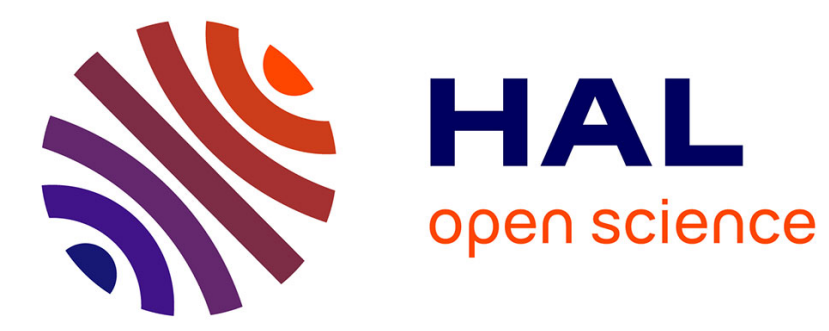

\title{
Microglial Priming as Trained Immunity in the Brain
}

Michael Haley, David Brough, Jessica Quintin, Stuart Allan

\section{To cite this version:}

Michael Haley, David Brough, Jessica Quintin, Stuart Allan. Microglial Priming as Trained Immunity in the Brain. Neuroscience, 2019, 405, pp.47-54. 10.1016/j.neuroscience.2017.12.039 . pasteur02873942

\section{HAL Id: pasteur-02873942 \\ https://hal-pasteur.archives-ouvertes.fr/pasteur-02873942}

Submitted on 23 Jun 2020

HAL is a multi-disciplinary open access archive for the deposit and dissemination of scientific research documents, whether they are published or not. The documents may come from teaching and research institutions in France or abroad, or from public or private research centers.
L'archive ouverte pluridisciplinaire HAL, est destinée au dépôt et à la diffusion de documents scientifiques de niveau recherche, publiés ou non, émanant des établissements d'enseignement et de recherche français ou étrangers, des laboratoires publics ou privés.

\section{(ㄷ)(1) $\$$}

Distributed under a Creative Commons Attribution - NonCommercial| 4.0 International 


\section{Microglial priming as trained immunity in the brain}

Michael J Haley ${ }^{1}$, David Brough ${ }^{1 *}$, Jessica Quintin ${ }^{2}$ and Stuart M. Allan ${ }^{1}$

${ }^{1}$ Division of Neuroscience and Experimental Psychology, Faculty of Biology, Medicine and Health, Manchester Academic Health Science Centre, The University of Manchester, Manchester, M13 9PT, UK.

${ }^{2}$ Immunology of Fungal Infections Group, Department of Mycology, Institut Pasteur, 25 rue du Docteur Roux, Paris, France.

${ }^{*}$ Corresponding author:

Dr David Brough

Division of Neuroscience and Experimental Psychology, Faculty of Biology, Medicine and Health, The University of Manchester, AV Hill Building Manchester, M13 9PT, UK Tel: +441612755039 Email: david.brough@manchester.ac.uk 


\section{Abstract}

In this review we discuss the possibility that the phenomenon of microglial priming can be explained by the mechanisms that underlie trained immunity. The latter involves the enhancement of inflammatory responses by epigenetic mechanisms that are mobilised after first exposure to an inflammatory stimulus. These mechanisms include long-lasting histone modifications, including H3K4me1 deposition at latent enhancer regions. Although such changes may be beneficial in peripheral infectious disease, in the context of microglial priming they may drive increased microglia reactivity that is damaging in diseases of brain ageing. 


\section{Introduction}

Exposure of microglia, the innate immune cell of the brain, to noxious or inflammatory stimuli induces a long lasting change, or memory, such that when the microglia encounter a subsequent inflammatory stimulus they produce a heightened inflammatory response. This is a phenomenon commonly described as microglial priming (Perry and Holmes, 2014). The initial stimulus could be a systemic illness or infection, with or without a central injury or insult, and is known to modify subsequent responses to disease and injury and affect disease outcome. Knowledge of the molecular mechanisms underpinning priming remains incomplete.

'Trained immunity' is an emerging concept that has been described mainly in peripheral innate immune cells and refers to the ability of such cells (for example, monocytes and tissue-resident macrophages) to develop and display a 'memory' to inflammatory and infectious challenges through epigenetic reprogramming; this process may explain the non-specific protective effect of some vaccines (Netea et al., 2016).

In this article, we speculate that the mechanisms underpinning trained immunity may explain microglial priming and offer new insights into how such priming is regulated in the brain.

\section{Trained immunity}

It was originally thought that the adaptive immune system builds up immunological memory but that the innate immune system is unable to do so. Recent findings show that this dichotomy is incorrect. Studies over the past decade have highlighted that innate immune cells - such as natural killer (NK) cells, monocytes and macrophages - are capable of remembering a first encounter with a pathogen, demonstrating that the non-specific innate immune system indeed has a memory. Whereas adaptive immune memory is characterised by genetic reprogramming in B-and T-cells, innate immune memory is instead mediated by epigenetic processes and regulation of transcriptions factors (Netea et al., 2016). This innate immune system memory means that innate immune cells are capable of a differential immune response - a heightened or tolerized immune response - following a secondary infection (Hamon and Quintin, 2016). This capacity of mammalian innate immune cells to recall a 
previous encounter and respond with an increased inflammatory response has been

Trained immunity associated with bacteria was first observed in human volunteers vaccinated with the mycobacterial Bacille Calmette-Guérin vaccine (BCG) (Kleinnijenhuis et al., 2012). In this study, BCG induced non-specific protective responses against non-mycobacterial challenges (for example, Candida albicans, Staphylococcus aureus and Escherichia coli) through functional reprogramming of innate immune monocytes. Such beneficial and protective non-specific effects of BCG had been seen before in epidemiological studies in BCG-vaccinated young West-African children (Garly et al., 2003), and also in experimental animals (van 't Wout et al., 1992). Newborn children vaccinated with BCG showed less susceptibility to infections other than tuberculosis, with a better overall survival in early childhood (Benn et al., 2013; Garly et al., 2003).

The past few years have seen an increasing interest in trained immunity and its underlying mechanisms. Using a lethal systemic infection model of murine 
candidiasis, a study showed that mice defective in functional $\mathrm{T}$ and $\mathrm{B}$ lymphocytes to infectious diseases but also to metabolic or non-communicable diseases. Indeed, the application of $\beta$-glucan to primary human monocytes induces the enrichment of $\mathrm{H} 3 \mathrm{~K} 4 \mathrm{me} 3$ at the promoters of genes relevant to atherosclerosis (Kleinnijenhuis et al., 2012) and increases the capacity of these cells to engulf oxidized low-density lipoprotein (oxLDL) and form foam cells (Bekkering et al., 2014). Interestingly, in vivo studies in cholesterol-fed New-Zealand white rabbits show that BCG immunization enhances peripheral leukocyte activation, aortic monocyte recruitment and atherogenesis (Lamb et al., 1999). Although one cannot exclude a contribution of the adaptive immune system to the data in these experiments, these findings fit the concept that trained immunity contributes to atherogenesis.

Trained immunity is probably induced not only by microbial products but also by metabolites such as damage-associated molecular patterns (DAMPs), which are relevant in the development of metabolic diseases and their associated complications. Human monocytes treated with the DAMPs oxLDL or acetylated LDL 
show long-term pro-inflammatory cytokine production and foam cell formation.

\section{Microglial activation and phenotype}

It is now recognised that microglia are capable of an extraordinary repertoire of actions and that, as such, microglial cell activity is tightly regulated. Alterations in brain homeostasis that result from ageing, infection, injury or disease affect the regulatory networks that control microglial function, leading to a change in phenotype of these cells (Perry and Holmes, 2014). This change in phenotype is frequently referred to as microglial 'activation', typically taken to represent 'classical' activation of the cells similar to what is observed in macrophages in response to bacterial or viral infection, characterised, amongst other things, by increased production of proinflammatory cytokines such as IL-1 (Girard et al., 2013). Depending on the stimulus microglia can also show alternative activation, characterised by expression of Arginase1 and chitinase-like molecule (YM1), amongst other things (Girard et al., 2013). 'Activated' microglia also show morphological changes, displaying shorter and thicker processes (Kreutzberg, 1996). Use of the term 'activated' in the context of microglia can however be misleading, since these cells are 'active' in their normal steady state; that is, they constantly monitor their environment and make frequent contact with neurons and other CNS-resident cells (Kettenmann et al., 2011; Nimmerjahn et al., 2005). Microglia are maintained in this mature steady-state by a range of factors within the CNS microenvironment (Perry and Holmes, 2014). For example, the interaction between microglial CX3CR1 receptors with constitutively expressed neuronal CX3CL1 acts as an inhibitory signal for microglial activation (Cardona et al., 2006). Similarly, astrocyte-secreted factors such as CSF-1 and TGF$\beta$ maintain microglia in their mature, monitoring state (Bohlen et al., 2017; Butovsky et al., 2014; Schilling et al., 2001). 
Microglia do not exist as one homogeneous population, but instead show considerable functional heterogeneity under both homeostatic and non-homeostatic (injury and disease) conditions (Gertig and Hanisch, 2014). For example, across specific regions of the brain microglia show different transcriptional profiles that change differentially with aging (Grabert et al., 2016). This heterogeneity of CNS microglia has been best explored by pioneering efforts to characterise microglia populations with single-cell resolution. Using unbiased computational analysis of single-cell transcriptomics or individual cell surface markers, distinct and previously unknown subpopulations of microglia have been identified in both the naïve and diseased brain (Keren-Shaul et al., 2017; Korin et al., 2017). Although the functional role of some of these subpopulations remains to be elucidated, some may have a role in disease. For example, Keren-Shaul and colleagues have identified a novel protective microglial subpopulation associated specifically with neurodegenerative disease (Keren-Shaul et al., 2017). These studies also highlight that categorisation of microglial activation using the M1-M2 spectrum is an oversimplification (Ransohoff, 2016). Ongoing research using similarly sensitive techniques to isolate and define microglial populations will likely reveal even further complexity.

\section{Microglial priming}

One recognised change in microglial phenotype is that of 'priming', whereby after experiencing an initial stimulus microglial cells show an exaggerated inflammatory response to a second stimulus. This phenomenon is thought to be the result of repeat or persistent exposure of microglia to inflammatory mediators, misfolded proteins or neuronal debris that occurs during ageing and neuropathological disease (Wes et al., 2016). Microglial priming was first demonstrated experimentally in mice with prion disease, as diseased mice showed a heightened microglial inflammatory response after peripheral or central LPS administration compared to naive mice (Cunningham, 2005). Similar findings of microglial priming have been reported after systemic infection with live bacteria (Püntener et al., 2012). The initial priming stimuli and secondary challenge may be temporally separated, for example inflammatory challenge in utero can lead to alterations in microglial reactivity later in life for offspring (Knuesel et al., 2014). Subsequent studies demonstrated that microglial priming can also be triggered by chronic stimuli, including ageing, stress, and neurodegeneration (Norden et al., 2015). Regardless of the initial priming stimuli, 
microglial priming is generally considered a maladaptive response. Microglial priming due to infection or LPS challenge results in a heightened sickness response to subsequent challenge (Perry and Holmes, 2014). In the context of ageing and neurodegeneration, microglial priming is associated with the development of cognitive deficits, impaired synaptic plasticity and accelerated neurodegeneration (Norden et al., 2015).

\section{Mechanisms of microglial priming}

Despite its clear contribution to pathology in several neurological conditions, the molecular pathways responsible for microglial priming remain only partially defined. In order to identify common gene networks that might underpin microglial priming, the microglial transcriptome was compared between conditions with known microglial priming, namely, mouse models of accelerated ageing, Alzheimer's disease and amyotrophic lateral sclerosis (Holtman et al., 2015). This comparison revealed gene expression networks common to these conditions thought to mediate microglial priming, including immune-, phagosome-, lysosome-, oxidative phosphorylation, and antigen presentation signalling pathways (Holtman et al., 2015). These pathways showed some overlap with pathways involved in general immune activation, for example IL-1 $\beta$ upregulation, but were distinct from pathways activated by acute immune challenge with LPS (Holtman et al., 2015). Increased microglial expression of $\mathrm{IL}-1 \beta$ as a feature of priming has also been reported in ageing, prion disease, in utero LPS exposure, and after repeat systemic inflammatory challenge (Cao et al., 2015; Cunningham, 2005; Field et al., 2010; Godbout et al., 2005; Hickman et al., 2013; Wynne et al., 2010). Increased antigen presentation, as measured by increased expression of MHC-II, also appears to be a common feature of microglial priming (Holtman et al., 2015; Perry and Holmes, 2014; Wynne et al., 2010), and is also seen in a small subset of microglia in naïve mice (Korin et al., 2017). Finally, release of microglia from inhibitory signals within the CNS, such as reduced CX3CR1-CX3CL1 signalling, also appears to be a common mediator of microglial priming (Cardona et al., 2006; Holtman et al., 2015; Wynne et al., 2010).

The identification of some common mechanisms and gene networks has gone some way to describing the transcriptional and inflammatory phenotype of primed microglia. However, without a more detailed understanding of the molecular 
pathways responsible for microglial priming, it will continue to be defined operationally (Perry and Holmes, 2014). Open questions include what mechanisms entrain the microglial inflammatory response over long periods, effectively storing the memory of their previous encounters with inflammatory stimuli. We suggest that this problem may be best studied by viewing microglial priming as trained immunity in the brain. We will therefore provide evidence that the molecular mechanisms that drive trained immunity also exist in microglia, and that these mechanisms could account for the experimental observations of microglial priming.

\section{Histone modifications in trained immunity and microglial priming}

The rate of transcription of genes can be affected by histone post-translational modifications (PTMs) at gene promoters and distal regulatory elements. Histone PTMs can negatively and positively affect transcription, and include methylation, acetylation, phosphorylation, ubiquitination (Lawrence et al., 2016). These modifications can be broadly classified as activating or repressing, leading to an increased propensity for open or closed chromatin, respectively (Lawrence et al., 2016; Venkatesh and Workman, 2015). The longevity of PTMs depends on the rate of histone turnover and the activity of PTM-removing enzymes (e.g. histone deacetylases and demethylases).

Acetylation occurs at the protruding $\mathrm{N}$-terminal tail of histones, and results in more relaxed chromatin, and so an increased propensity for gene transcription. The degree of histone acetylation is mediated by the balance between enzymes which attach or remove acetyl groups, namely histone acetyltransferases (HATs) and histone deacetylases (HDACs). Pharmacological HDAC inhibitors have been shown to have an anti-inflammatory action in vitro and in vivo in several CNS pathologies, supposedly by preventing the inhibition of anti-inflammatory genes (Kaminska et al., 2016). This has mostly been investigated at the level of the acute inflammatory response, for example HDAC inhibition reduces LPS-mediated expression of cytokines in microglia (Durham et al., 2017; Singh et al., 2014). However, treatments proposed to act through HDAC inhibition can also promote a ramified morphology in microglia usually associated with a quiescent or non-activated phenotype (Huang et al., 2017). 
Next generation sequencing technologies such as CHIP-seq and ATAC-seq have begun to allow an exploration of epigenetic phenotype of microglia, and the genomewide deposition of specific histone modifications (Gosselin et al., 2014; Gosselin et al., 2017). These studies have established that there is an important interplay between epigenetic phenomena (specifically, H3K4me2 and H3K27ac deposition) and transcription factors in determining microglial differentiation and function (Gosselin et al., 2014; Gosselin et al., 2017). Evidence that histone modifications could drive microglial priming in disease comes from work of Keren-Shaul and colleagues, who explored the epigenetic profile of microglia during Alzheimer's disease (Keren-Shaul et al., 2017). They measured the genome-wide distribution of histone 3 lysine 4 dimethylation (H3K4me2), a methylation PTM known to mark promoter and enhancer regions, in microglia isolated from wildtype and genetic Alzheimer's disease mice. In this study they identified a novel microglial phenotype associated with plaques and disease progression they termed disease associated microglia (DAM). When comparing the H3K4me2 read coverage between non-DAM and DAM microglia within Alzheimer mice they found that there was little difference, however there were evident differences between WT and Alzheimer microglia. This suggests that epigenetic changes occurring during Alzheimer's disease are priming microglia for a later transition to the DAM phenotype (Keren-Shaul et al., 2017). These studies clearly demonstrate that histone modifications are important in determining microglial lineage and phenotype, however there is a lack of evidence for an epigenetic mechanism that recapitulates the canonical finding of microglial priming; a heightened inflammatory response upon re-challenge with a stimuli. However, the epigenetic mechanisms that mediate trained immunity in macrophages are better understood, focusing on the role of de novo or latent enhancers. Latent enhancers are genomic regions unbound by transcription factors and with few histone modifications in unstimulated macrophages, but that then acquire epigenetic modifications that allow for a stronger response upon re-challenge with similar stimuli (Ostuni et al., 2013; Saeed et al., 2014). These epigenetic changes are long-lasting, and characterised by H3K4me1 deposition. If the mechanisms of trained immunity and microglial priming were to overlap, we would predict that H3M4me1 deposition would play a similar role, potentially mediating the transcriptional phenotype associated with microglial priming (Holtman et al., 2015). However, the role of H3M4me1 deposition in microglia is currently unexplored. 


\section{Epigenetic regulation of IL-1 in microglia}

Inflammatory mediators of the IL-1 family have been particularly implicated in brain disease, including Alzheimer's disease and stroke (Allan et al., 2005). The IL-1 ligand super family comprises 11 cytokines of which the best-defined and most potent pro-inflammatory forms are IL-1 $\alpha$ and IL-1 $\beta$ (Dinarello, 2013). Both are produced by microglia as precursors that require proteolytic conversion to active secreted forms which is facilitated by multi-molecular complexes called inflammasomes. As discussed, increased microglial IL-1 expression upon exposure to subsequent inflammatory stimuli is a common feature of microglial priming in several conditions (Cao et al., 2015; Cunningham, 2005; Field et al., 2010; Godbout et al., 2005; Hickman et al., 2013; Holtman et al., 2015; Wynne et al., 2010). Recent evidence suggests that IL-1 and related genes are epigenetically regulated in microglia (Cho et al., 2015; Matt et al., 2016), suggesting that epigenetic mechanisms are important in both trained immunity and microglial priming. In microglia from the brains of elderly people, and people with dementia, the proximal promoter of IL1B (which encodes IL-1 $\beta$ ) is hypomethylated at two CpG sites, resulting in increased IL-1 $\beta$ expression (Cho et al., 2015). Another group found that expression of DNA methylating enzymes is decreased in microglia from aged mice and that this decrease is associated with decreased methylation of the ILIB promoter which results in increased IL-1 production and exaggerated sickness behaviour responses (Matt et al., 2016). IL-1 family and inflammasome-associated genes are also subject to epigenetic modification in cells from patients with cryopyrin-associated periodic syndromes, conditions characterised by IL-1 $\beta$ mediated systemic inflammation (Vento-Tormo et al., 2017). Together, these data demonstrate that inflammatory pathways implicated in microglial priming may be epigenetically regulated. We anticipate that the application of epigenetic profiling in CNS disease will reveal that other important inflammatory pathways within microglia are also under epigenetic regulation.

\section{Microglial priming in the context of the microglial life cycle}

We have suggested that microglial priming could be mediated by epigenetic mechanisms similar to those identified in trained immunity in other innate immune cells. However, an important difference between microglia and other innate immune 
cells is that microglia are longer-lived, proliferating at a rate 20 fold less than other tissue resident macrophages (Lawson et al., 1992). During the steady-state, microglia renew and replace themselves by proliferation without contribution from circulating progenitors, with the overall number of microglia remaining constant due to a coupling between proliferation and apoptosis (Askew et al., 2017). Newly dividing microglia disperse to adopt their own territory (Askew et al., 2017; Tay et al., 2017), integrating into the microglial network. Estimates of the rate of this process of microglial turn-over have varied, with several recent studies attempting to improve on older estimates made using autoradiography, which estimated that $0.05 \%$ of microglia were dividing at any one time (Lawson et al., 1992). Assuming that the innate immune memory of microglia is not in some way heritable to daughter cells produced during clonal expansion, the actual rate of microglia turn-over is likely to be an important determinant of how long microglial priming effects could persist. For example, Askew and colleagues found that on average $0.69 \%$ of microglia were proliferating at any one time, suggesting that the entire microglial population in mice may be replaced every 96 days; several times over an animals lifetime (Askew et al., 2017). This may place an upper limit on how long priming effects may last. However, other studies reported microglial turn-over to be more similar to original estimates made by Lawson and colleagues, suggesting that at the whole population level microglia are very long-lived. A study in humans identified a median microglial age of 4.2 years, with some microglia living as long as 20 years (Réu et al., 2017). A similar estimation in mice suggested a median microglial life-span of 15 months (Füger et al., 2017). However, at the single-cell level there appears to be considerable heterogeneity in microglial age (Tay et al., 2017), which increases with ageing (Füger et al., 2017). Differing rates of proliferation have also been reported between different brain regions (Askew et al., 2017; Tay et al., 2017), and the rates of apoptosis appear to be higher in newly-divided compared to existing microglia (Askew et al., 2017). These data suggest that although microglia are long-lived during their steady-state, they are not uniformly so, and that subpopulations of microglia may divide more rapidly. In contrast to the steady-state, CNS injury is robustly associated with a rapid increase in the number and density of microglia, driven partially by microglial migration from distant sites, but also by microglial proliferation (Gomez-Nicola and Perry, 2015; Tay et al., 2017). This proliferative response is driven by selected clonal expansion at the injury site (Tay et al., 2017). If 


\section{Conclusions}

The initial reports of trained immunity describe it as an evolutionary beneficial response to mount more effective responses to pathogen after subsequent infections

We propose a model of a model of microglial priming driven by latent enhancers, as found within trained immunity in macrophages, would be consistent with the aforementioned findings in microglia (Figure 1). The initial CNS injury drives a local accumulation of microglia, and promotes microglial activation. The resulting transcriptomic phenotype of these activate microglia will include inflammatory pathways common to many stimuli, but may also feature pathways specific to the stimuli encountered. After a period of overt microglial activation, so long as the CNS insult is resolved and the activating stimuli removed, the remaining microglia will eventually adopt their steady-state phenotype (Tay et al., 2017). However, memory of this encounter may be stored as H3K4me1 deposition at specific sites in the microglial genome. H3K4me1 modifications indicate enhancers in a poised state which allow an augmented response upon re-counter with the same stimuli (Ostuni et al., 2013; Saeed et al., 2014). Importantly, H3K4me1 modifications are longlasting, persisting even when the initial stimuli is removed (Ostuni et al., 2013). Microglial phenotype is also modulated by other epigenetic factors, including H3K4me2 and H3K27ac deposition (Gosselin et al., 2014; Gosselin et al., 2017). Both these epigenetic changes and the microglia themselves have been shown to be very long-lived, allowing a long-lasting memory of the initial encounter. Subsequent re-encounter with similar stimuli would lead to a heightened response due to genetic pathways being already primed for activation by epigenetic markers. Importantly, these mechanisms could allow microglia to remember previous stimuli (as has been observed experimentally in microglia priming) without interfering with microglia eventually returning to a steady-state. However, we could speculate that an overaccumulation of epigenetic changes may eventually contribute to pathology by tipping the balance towards a state of hyper-sensitivity or persistent activation, as is often found in neurodegenerative conditions. 
(Bistoni et al., 1986; Kleinnijenhuis et al., 2012). However, trained immunity also occurs in non-communicable diseases, with the best evidence to-date suggested from studies on atherogenic disease (Bekkering et al., 2014; Lamb et al., 1999). Here, and in other diseases of old age such as stroke and Alzheimer's disease, rather than being a beneficial response, trained immunity probably represents a maladaptive response that exacerbates damaging inflammatory mechanisms, and it occurs in old age owing to a lack of evolutionary pressure to select against it. There are striking parallels between trained immunity in cells of the peripheral innate immune system, and microglial priming in the brain. Given evidence of long-term innate immune memory in microglia, microglial longevity, and the existence of epigenetic mechanisms modulating inflammatory pathways in microglia, we speculate that the underlying mechanisms of microglial priming may be closely related to trained immunity. Future studies combining epigenetic approaches with next generation sequencing in microglia will broaden our understanding in this area, potentially identifying causal relationships between epigenetic phenomena and CNS disease. 


\section{Figure Legend}

Figure 1: A proposed model of microglial priming and innate immune memory based on trained immunity in tissue-resident macrophages. Microglia are maintained in their steady state by inhibitory signals, however encountering various stimuli can lead to a phenotypic change commonly referred to as microglial activation. Once the activating stimulus is removed, microglia can return to their steady-state. However, an epigenetic memory of the stimuli may be stored in histone modifications, specifically by H3K4me1 deposition at defined latent enhancer sites in the genome. These latent enhancers may contribute to the augmented response upon re-encounter with similar stimuli that has been reported experimentally in models of microglial priming. Microglial activation can also result in other histone modifications, including deposition of H3K4me2, H3K4me3 and H3K27ac. It may be possible that repeated exposure to inflammatory stimuli leads to an accumulation of epigenetic changes that lock microglia in a hyper-reactive phenotype, as is found in many neurodegenerative conditions. 


\section{References}

Allan, S. M., Tyrrell, P. J. and Rothwell, N. J. (2005). Interleukin-1 and neuronal injury. Nat. Rev. Immunol. 5, 629-640.

Askew, K., Li, K., Olmos-Alonso, A., Garcia-Moreno, F., Liang, Y., Richardson, P., Tipton, T., Chapman, M. A., Riecken, K., Beccari, S., et al. (2017). Coupled Proliferation and Apoptosis Maintain the Rapid Turnover of Microglia in the Adult Brain. Cell Rep. 18, 391-405.

Bekkering, S., Quintin, J., Joosten, L. A. B., Van Der Meer, J. W. M., Netea, M. G. and Riksen, N. P. (2014). Oxidized low-density lipoprotein induces long-term proinflammatory cytokine production and foam cell formation via epigenetic reprogramming of monocytes. Arterioscler. Thromb. Vasc. Biol. 34, 1731-1738.

Benn, C. S., Netea, M. G., Selin, L. K. and Aaby, P. (2013). A Small Jab - A Big Effect: Nonspecific Immunomodulation By Vaccines. Trends Immunol. 34, 431439.

Bistoni, F., Vecchiarelli, A., Cenci, E., Puccetti, P., Marconi, P. and Cassone, A. (1986). Evidence for macrophage-mediated protection against lethal Candida albicans infection. Infect. Immun. 51, 668-674.

Bistoni, F., Verducci, G., Perito, S., Vecchiarelli, A., Puccetti, P., Marconi, P. and Cassone, A. (1988). Immunomodulation by a low-virulence, agerminative variant of candida albicans. Further evidence for macrophage activation as one of the effector mechanisms of nonspecific anti-infectious protection. Med. Mycol. 26, 285-299.

Biswas, S. K. and Mantovani, A. (2010). Macrophage plasticity and interaction with lymphocyte subsets: cancer as a paradigm. Nat. Immunol. 11, 889-896.

Bohlen, C. J., Bennett, F. C., Tucker, A. F., Collins, H. Y., Mulinyawe, S. B. and Barres, B. A. (2017). Diverse Requirements for Microglial Survival, Specification, and Function Revealed by Defined-Medium Cultures. Neuron 94 , 759-773.e8.

Bowdish, D. M. E., Loffredo, M. S., Mukhopadhyay, S., Mantovani, A. and Gordon, S. (2007). Macrophage receptors implicated in the "adaptive" form of innate immunity. Microbes Infect. 9, 1680-1687.

Butovsky, O., Jedrychowski, M. P., Moore, C. S., Cialic, R., Lanser, A. J., Gabriely, G., Koeglsperger, T., Dake, B., Wu, P. M., Doykan, C. E., et al. (2014). Identification of a unique TGF- $\beta$-dependent molecular and functional 
signature in microglia. Nat. Neurosci. 17, 131-43.

Cao, M., Cortes, M., Moore, C. S., Leong, S. Y., Durosier, L. D., Burns, P., Fecteau, G., Desrochers, A., Auer, R. N., Barreiro, L. B., et al. (2015). Fetal microglial phenotype in vitro carries memory of prior in vivo exposure to inflammation. Front. Cell. Neurosci. 9,.

Cardona, A. E., Pioro, E. P., Sasse, M. E., Kostenko, V., Cardona, S. M., Dijkstra, I. M., Huang, D., Kidd, G., Dombrowski, S., Dutta, R., et al. (2006). Control of microglial neurotoxicity by the fractalkine receptor. Nat. Neurosci. $\mathbf{9}$, 917-924.

Cheng, S.-C., Quintin, J., Cramer, R. A., Shepardson, K. M., Saeed, S., Kumar, V., Giamarellos-Bourboulis, E. J., Martens, J. H. A., Rao, N. A., Aghajanirefah, A., et al. (2014). mTOR- and HIF-1a-mediated aerobic glycolysis as metabolic basis for trained immunity. Science 345, 1250684.

Cho, S.-H., Chen, J. A., Sayed, F., Ward, M. E., Gao, F., Nguyen, T. A., Krabbe, G., Sohn, P. D., Lo, I., Minami, S., et al. (2015). SIRT1 Deficiency in Microglia Contributes to Cognitive Decline in Aging and Neurodegeneration via Epigenetic Regulation of IL-1 . J. Neurosci. 35, 807-818.

Cunningham, C. (2005). Central and Systemic Endotoxin Challenges Exacerbate the Local Inflammatory Response and Increase Neuronal Death during Chronic Neurodegeneration. J. Neurosci. 25, 9275-9284.

Dinarello, C. A. (2013). Overview of the interleukin-1 family of ligands and receptors. Semin. Immunol. 25, 389-393.

Durham, B. S., Grigg, R. and Wood, I. C. (2017). Inhibition of histone deacetylase 1 or 2 reduces induced cytokine expression in microglia through a protein synthesis independent mechanism. J. Neurochem. 0-3.

Field, R., Campion, S., Warren, C., Murray, C. and Cunningham, C. (2010). Systemic challenge with the TLR3 agonist poly I: $C$ induces amplified IFNa/ $\beta$ and IL-1 $\beta$ responses in the diseased brain and exacerbates chronic neurodegeneration. Brain. Behav. Immun. 24, 996-1007.

Füger, P., Hefendehl, J. K., Veeraraghavalu, K., Wendeln, A.-C., Schlosser, C., Obermüller, U., Wegenast-Braun, B. M., Neher, J. J., Martus, P., Kohsaka, S., et al. (2017). Microglia turnover with aging and in an Alzheimer's model via long-term in vivo single-cell imaging. Nat. Neurosci.

Garly, M. L., Martins, C. L., Balé, C., Baldé, M. A., Hedegaard, K. L., Gustafson, 
P., Lisse, I. M., Whittle, H. C. and Aaby, P. (2003). BCG scar and positive tuberculin reaction associated with reduced child mortality in West Africa: A nonspecific beneficial effect of BCG? Vaccine 21, 2782-2790.

Gertig, U. and Hanisch, U.-K. (2014). Microglial diversity by responses and responders. Front. Cell. Neurosci. 8,.

Girard, S., Brough, D., Lopez-Castejon, G., Giles, J., Rothwell, N. J. and Allan, S. M. (2013). Microglia and macrophages differentially modulate cell death after brain injury caused by oxygen-glucose deprivation in organotypic brain slices. Glia 61, 813-24.

Godbout, J. P., Chen, J., Abraham, J., Richwine, a F., Berg, B. M., Kelley, K. W. and Johnson, R. W. (2005). Exaggerated neuroinflammation and sickness behavior in aged mice following activation of the peripheral innate immune system. FASEB J. 19, 1329-31.

Gomez-Nicola, D. and Perry, V. H. (2015). Microglial Dynamics and Role in the Healthy and Diseased Brain: A Paradigm of Functional Plasticity. Neurosci. 21, 169-184.

Gosselin, D., Link, V. M., Romanoski, C. E., Fonseca, G. J., Eichenfield, D. Z., Spann, N. J., Stender, J. D., Chun, H. B., Garner, H., Geissmann, F., et al. (2014). Environment drives selection and function of enhancers controlling tissue-specific macrophage identities. Cell 159, 1327-1340.

Gosselin, D., Skola, D., Coufal, N. G., Holtman, I. R., Schlachetzki, J. C. M., Sajti, E., Jaeger, B. N., O?Connor, C., Fitzpatrick, C., Pasillas, M. P., et al. (2017). An environment-dependent transcriptional network specifies human microglia identity. Science (80-. ). 3222, eaal3222.

Grabert, K., Michoel, T., Karavolos, M. H., Clohisey, S., Baillie, J. K., Stevens, M. P., Freeman, T. C., Summers, K. M. and McColl, B. W. (2016). Microglial brain region-dependent diversity and selective regional sensitivities to aging. Nat. Neurosci. 19, 504-516.

Hamon, M. A. and Quintin, J. (2016). Innate immune memory in mammals. Semin. Immunol. 28, 351-358.

Hickman, S. E., Kingery, N. D., Ohsumi, T. K., Borowsky, M. L., Wang, L., Means, T. K. and El Khoury, J. (2013). The microglial sensome revealed by direct RNA sequencing. Nat. Neurosci. 16, 1896-1905.

Holtman, I. R., Raj, D. D., Miller, J. A., Schaafsma, W., Yin, Z., Brouwer, N., Wes, 
P. D., Möller, T., Orre, M., Kamphuis, W., et al. (2015). Induction of a common microglia gene expression signature by aging and neurodegenerative conditions: a co-expression meta-analysis. Acta Neuropathol. Commun. 3, 31.

Huang, C., Wang, P., Xu, X., Zhang, Y., Gong, Y., Hu, W., Gao, M., Wu, Y., Ling, Y., Zhao, X., et al. (2017). The ketone body metabolite $\beta$-hydroxybutyrate induces an antidepression-associated ramification of microglia via HDACs inhibition-triggered Akt-small RhoGTPase activation. Glia.

Kaminska, B., Mota, M. and Pizzi, M. (2016). Signal transduction and epigenetic mechanisms in the control of microglia activation during neuroinflammation. Biochim. Biophys. Acta - Mol. Basis Dis. 1862, 339-351.

Keren-Shaul, H., Spinrad, A., Weiner, A., Matcovitch-Natan, O., Dvir-Szternfeld, R., Ulland, T. K., David, E., Baruch, K., Lara-Astaiso, D., Toth, B., et al. (2017). A Unique Microglia Type Associated with Restricting Development of Alzheimer's Disease. Cell 169, 1276-1290.e17.

Kettenmann, H., Hanisch, U. K., Noda, M. and Verkhratsky, A. (2011). Physiology of Microglia. Physiol. Rev. 91, 461-553.

Kleinnijenhuis, J., Quintin, J., Preijers, F., Joosten, L. A. B., Ifrim, D. C., Saeed, S., Jacobs, C., van Loenhout, J., de Jong, D., Stunnenberg, H. G., et al. (2012). Bacille Calmette-Guerin induces NOD2-dependent nonspecific protection from reinfection via epigenetic reprogramming of monocytes. Proc. Natl. Acad. Sci. 109, 17537-17542.

Knuesel, I., Chicha, L., Britschgi, M., Schobel, S. A., Bodmer, M., Hellings, J. A., Toovey, S. and Prinssen, E. P. (2014). Maternal immune activation and abnormal brain development across CNS disorders. Nat. Rev. Neurol. 10, 643660 .

Korin, B., Ben-Shaanan, T. L., Schiller, M., Dubovik, T., Azulay-Debby, H., Boshnak, N. T., Koren, T. and Rolls, A. (2017). High-dimensional, single-cell characterization of the brain's immune compartment. Nat. Neurosci.

Kreutzberg, G. W. (1996). Microglia: A sensor for pathological events in the CNS. Trends Neurosci. 19, 312-318.

Lamb, D. J., Eales, L. J. and Ferns, G. A. (1999). Immunization with bacillus Calmette-Guerin vaccine increases aortic atherosclerosis in the cholesterol-fed rabbit. Atherosclerosis 143, 105-113.

Lawrence, M., Daujat, S. and Schneider, R. (2016). Lateral Thinking: How Histone 
Modifications Regulate Gene Expression. Trends Genet. 32, 42-56.

Lawson, L. J., Perry, V. H. and Gordon, S. (1992). Turnover of resident microglia in the normal adult mouse brain. Neuroscience 48, 405-415.

Matt, S. M., Lawson, M. A. and Johnson, R. W. (2016). Aging and peripheral lipopolysaccharide can modulate epigenetic regulators and decrease IL-1 $\beta$ promoter DNA methylation in microglia. Neurobiol. Aging 47, 1-9.

Min-Oo, G. and Lanier, L. L. (2014). Cytomegalovirus generates long-lived antigenspecific NK cells with diminished bystander activation to heterologous infection. J. Exp. Med. 211, 2669-2680.

Netea, M. G., Quintin, J. and Van Der Meer, J. W. M. (2011). Trained immunity: A memory for innate host defense. Cell Host Microbe 9, 355-361.

Netea, M. G., Joosten, L. A. B., Latz, E., Mills, K. H. G., Natoli, G., Stunnenberg, H. G., ONeill, L. A. J. and Xavier, R. J. (2016). Trained immunity: A program of innate immune memory in health and disease. Science (80-. ). 352, aaf1098aaf1098.

Nimmerjahn, A., Kirchhoff, F. and Helmchen, F. (2005). Resting microglial cells are highly dynamic surveillants of brain parenchyma in vivo. Neuroforum 11, 9596.

Norden, D. M., Muccigrosso, M. M. and Godbout, J. P. (2015). Microglial priming and enhanced reactivity to secondary insult in aging, and traumatic CNS injury, and neurodegenerative disease. Neuropharmacology 96, 29-41.

O'Leary, J. G., Goodarzi, M., Drayton, D. L. and von Andrian, U. H. (2006). T cell- and B cell-independent adaptive immunity mediated by natural killer cells. Nat. Immunol. 7, 507-516.

O'Sullivan, T. E., Sun, J. C. and Lanier, L. L. (2015). Natural Killer Cell Memory. Immunity 43, 634-645.

Ostuni, R., Piccolo, V., Barozzi, I., Polletti, S., Termanini, A., Bonifacio, S., Curina, A., Prosperini, E., Ghisletti, S. and Natoli, G. (2013). Latent enhancers activated by stimulation in differentiated cells. Cell 152, 157-171.

Paust, S., Gill, H. S., Wang, B.-Z., Flynn, M. P., Moseman, E. A., Senman, B., Szczepanik, M., Telenti, A., Askenase, P. W., Compans, R. W., et al. (2010). Critical role for the chemokine receptor CXCR6 in NK cell-mediated antigenspecific memory of haptens and viruses. Nat. Immunol. 11, 1127-1135.

Perry, V. H. and Holmes, C. (2014). Microglial priming in neurodegenerative 
disease. Nat. Rev. Neurol. 10, 217-224.

Püntener, U., Booth, S. G., Perry, V. H. and Teeling, J. L. (2012). Long-term impact of systemic bacterial infection on the cerebral vasculature and microglia. J. Neuroinflammation 9, 668.

Ransohoff, R. M. (2016). A polarizing question: do M1 and M2 microglia exist? Nat. Neurosci. 19, 987-91.

Réu, P., Khosravi, A., Bernard, S., Mold, J. E., Salehpour, M., Alkass, K., Perl, S., Tisdale, J., Possnert, G., Druid, H., et al. (2017). The Lifespan and Turnover of Microglia in the Human Brain. Cell Rep. 20, 779-784.

Saeed, S., Quintin, J., Kerstens, H. H. D., Rao, N. A., Matarese, F., Cheng, S., Ratter, J., Ent, M. A. Van Der, Sharifi, N., Janssen-megens, E. M., et al. (2014). Epigenetic programming during monocyte to macrophage differentiation and trained innate immunity. Science (80-. ). 345, 1-26.

Schilling, T., Nitsch, R., Heinemann, U., Haas, D. and Eder, C. (2001). Astrocytereleased cytokines induce ramification and outward $\mathrm{K}+$ channel expression in microglia via distinct signalling pathways. Eur. J. Neurosci. 14, 463-473.

Singh, V., Bhatia, H. S., Kumar, A., de Oliveira, A. C. P. and Fiebich, B. L. (2014). Histone deacetylase inhibitors valproic acid and sodium butyrate enhance prostaglandins release in lipopolysaccharide-activated primary microglia. Neuroscience 265, 147-157.

Sun, J. C., Beilke, J. N. and Lanier, L. L. (2009). Adaptive immune features of natural killer cells. Nature 457, 557-561.

Sun, J. C., Beilke, J. N., Bezman, N. A. and Lanier, L. L. (2011). Homeostatic proliferation generates long-lived natural killer cells that respond against viral infection. J. Exp. Med. 208, 357-368.

Sun, J. C., Madera, S., Bezman, N. A., Beilke, J. N., Kaplan, M. H. and Lanier, L. L. (2012). Proinflammatory cytokine signaling required for the generation of natural killer cell memory. J. Exp. Med. 209, 947-954.

Tay, T. L., Mai, D., Dautzenberg, J., Fernández-Klett, F., Lin, G., Sagar, Datta, M., Drougard, A., Stempfl, T., Ardura-Fabregat, A., et al. (2017). A new fate mapping system reveals context-dependent random or clonal expansion of microglia. Nat. Neurosci. 20, 793-803.

van 't Wout, J. W., Poell, R. and van Furth, R. (1992). The role of BCG/PPDactivated macrophages in resistance against systemic candidiasis in mice. 
Scand. J. Immunol. 36, 713-9.

Vecchiarelli, A., Cenci, E., Puliti, M., Blasi, E., Puccetti, P., Cassone, A. and Bistoni, F. (1989). Protective immunity induced by low-virulence Candida albicans: Cytokine production in the development of the anti-infectious state. Cell. Immunol. 124, 334-344.

Venkatesh, S. and Workman, J. L. (2015). Histone exchange, chromatin structure and the regulation of transcription. Nat. Rev. Mol. Cell Biol. 16, 178-189.

Vento-Tormo, R., Álvarez-Errico, D., Garcia-Gomez, A., Hernández-Rodríguez, J., Buján, S., Basagaña, M., Méndez, M., Yagüe, J., Juan, M., Aróstegui, J. I., et al. (2017). DNA demethylation of inflammasome-associated genes is enhanced in patients with cryopyrin-associated periodic syndromes. J. Allergy Clin. Immunol. 139, 202-211.e6.

Wes, P. D., Holtman, I. R., Boddeke, E. W. G. M., Möller, T. and Eggen, B. J. L. (2016). Next generation transcriptomics and genomics elucidate biological complexity of microglia in health and disease. Glia 64, 197-213.

Wynne, A. M., Henry, C. J., Huang, Y., Cleland, A. and Godbout, J. P. (2010). Protracted downregulation of CX3CR1 on microglia of aged mice after lipopolysaccharide challenge. Brain. Behav. Immun. 24, 1190-1201. 


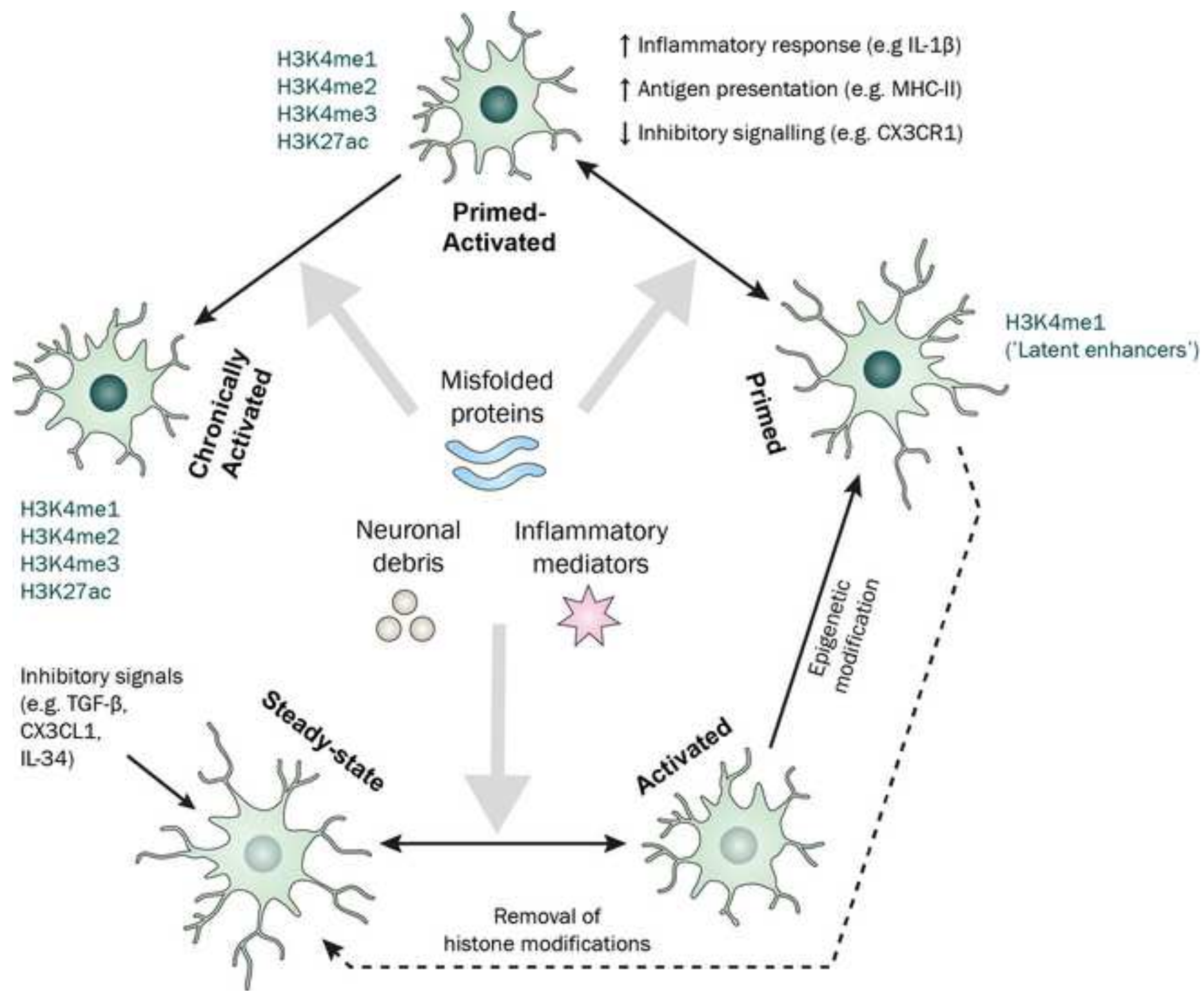

